\title{
Impossible primary referral to the major trauma center of an integrated trauma system: a case of exsanguinating trauma treated in a Spoke hospital
}

\author{
Emiliano Gamberini ${ }^{1}$, Antonella Potalivo ${ }^{2}$, Marco Benni ${ }^{1}$ and Vanni Agnoletti ${ }^{1}$ \\ ${ }^{1}$ Anesthesia and Intensive Care Unit, Emergency Department, Maurizio Bufalini Hospital, Italy \\ ${ }^{2}$ Anesthesia and Intensive Care Unit, Surgical Department, Infermi Hospital, Italy
}

\begin{abstract}
Background: Major trauma is a leading cause of death all over the world. Integrated trauma systems have been widely built up on a hub and spoke model by identification of a Major Trauma Centers (Hub) and secondary acute care hospital (Spokes) covering various population amount. Helicopter Emergency Medical System where involved in organization and learning programs. According to literature, primary referral to Major Trauma Centers of severely injured trauma victims, whenever possible, is associated with better outcomes. Trauma System in Emilia-Romagna region (Italy) is based on 3 Integrated Trauma Systems called SIAT with a Major Trauma Center each. Romagna-SIAT covers the east coast towards the Adriatic Sea, with an estimated population of 1.250.000.

Case presentation: We present a case in which massive bleeding by traumatic penetrating injury in zone 1 of the neck made impossible the primary referral to Major Trauma Center for clinical and logistical circumstances. The only available choice was to bring the patient to the nearest Spoke hospital where optimal interaction between Helicopter crew and Spoke hospital personnel, used working with preferred partners, was crucial for good patient outcome. Effective communications by phone during ambulance transport to the nearest hospital, not used to treat severe trauma cases, was effective in fulfilling lack of knowledge between team clinicians. Direct access to Operating Theater by-passing Emergency Department acceptance for surgical damage control of bleeding, together with Early Coagulative Support Protocol activation and hypotensive resuscitation strategy where coordinated allowing an updated and effective treatment.

Conclusions: Primary referral to Major Trauma Centers of severe trauma victims had shown to improve outcome. Dedicated Helicopter services are widely used to reduce transport time. In case of problems in airways magement or uncontrolled external hemorrhage, primary referral could lead to death during transport, instead of damage control treatment in the nearest Spoke hospital with Anesthesiologists and Surgeons 24/7. Spoke hospital performance in treating severe trauma victims could be critical because of the small volume of cases per year. We present a case in which effective planning, training and communication in an integrated trauma system allowed to get good clinical outcome.
\end{abstract}

\section{List of abbreviations}

MCT: Major Trauma Center; HEMS: Helicopter Medical Emergency System; GCS: Glasgow Coma Scale; SIAT: Integrated Trauma Assistance System; BP: Blood Pressure; SpO2: Peripheral Oxygen Saturation; OR: Operating Room.

\section{Introduction}

Major trauma is a leading cause of death all over the world. Comprehensive trauma networks, based on identification of Major Trauma Centers (MTCs) and secondary acute care hospital into a hub and spoke model, together with integrated prehospital care systems, are associated with improved outcomes [1,2]. Helicopter Emergency Medical System (HEMS) is internationally involved in trauma regional organization, with specific training of HEMS crews, according to the constitution of MTCs in-hospital Trauma Teams [3-5]. Trauma System in Emilia-Romagna region (Italy) is based on 3 Integrated Trauma Systems called SIAT with MTC each. Romagna-SIAT covers the east coast towards the Adriatic Sea and Romagna Region (Figure 1) with an estimated population of 1.2 million in 2016 that significantly increases during summer holydays [6].

\section{Case presentation}

A young man cyclist hit a neutral service vehicle during a local race. The car suddenly slowed and the cyclist was thrown into the car by the rear window. Bystanders extricated him and a severe bleeding from a penetrating injury of right zone 1 of the neck started [7]. Hemorrhage was suddenly controlled by direct compression while an ambulance and HEMS were on the way. Patient was found by ambulance's team on the road in shock with large amounts of blood on the ground. The direct compression of the neck wound prevents the cervical collar use and patient was loaded into the ambulance where supplementary oxygen was administrated, two large bore venous cannulas were positioned and crystalloids and colloids infusion started. 5 minutes later HEMS crew landed in a field 100 meters far from the scene. HEMS doctor

Correspondence to: Emiliano Gamberini, Anesthesia and Intensive Care Unit, Emergency Department, Maurizio Bufalini Hospital, Viale Ghirotti 286, zip code 47521, Cesena, Italy, Tel: +39 06 440611; E-mail: emiliano.gamberini@auslromagna.it

Key words: penetrating trauma, neck, shock, major trauma center, trauma system, trauma leader, hypotensive resuscitation

Received: March 16, 2017; Accepted: April 12, 2017; Published: April 14, 2017 


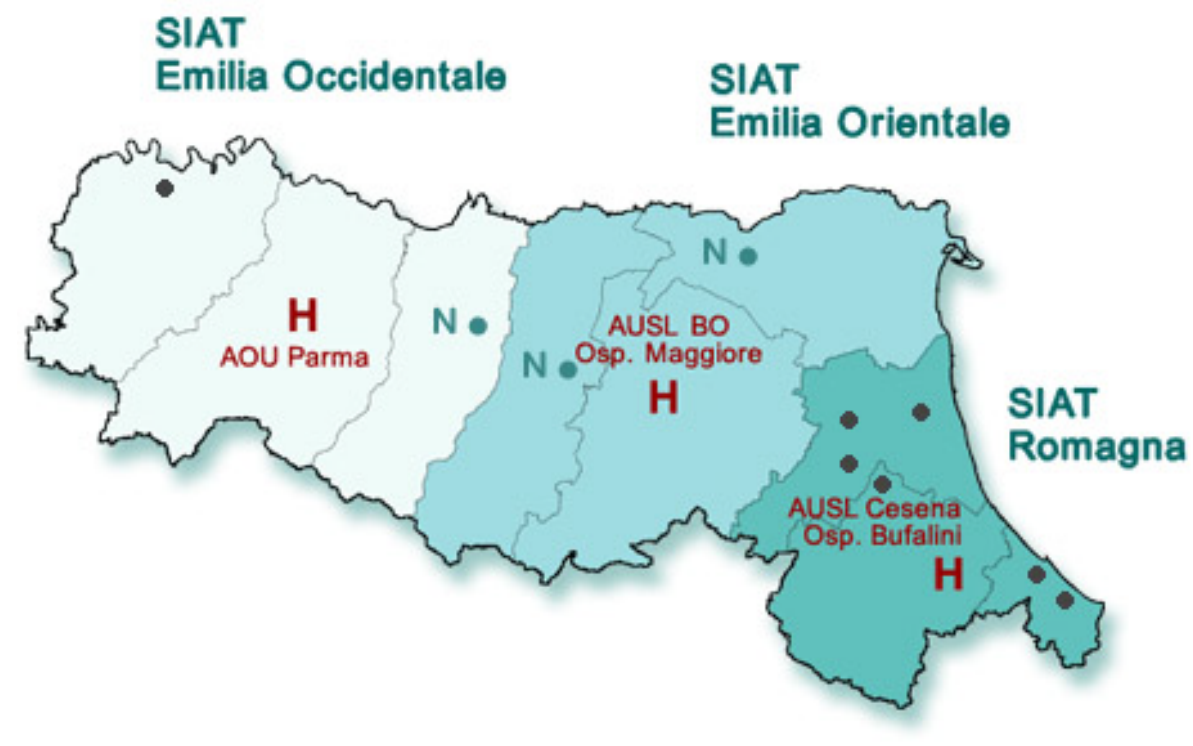

Figure 1. Emilia Romagna Trauma System with the 3 SIATs. H: Major Trauma Centers. N: spoke acute care hospitals with Neuro Surgery. Dark dots: secondary acute care hospitals.

primary survey revealed a Glasgow Coma Scale (GCS) of 3 with bilateral mydriasis, respiratory rate below $9 / \mathrm{min}$ with a peri-arrest clinical status. Tracheal intubation was performed without drugs requirement and mechanical ventilation started. Clinical conditions slightly improved with resolution of mydriasis, return of palpable left carotid pulse and the act of checking the left sited pulse induced transient left pupil dilation. Vesicular murmur was present bilaterally and pelvis was stable by clinical examination. Direct compression of the wound begun to be un-effective in controlling mixed venous and arterial bleeding. Using Foleys catheter balloon to close injured vassels was unfeasible for multiple vascular injuries. After infusing crystalloids $250 \mathrm{ml}$ and colloids $250 \mathrm{ml}$ intravenous therapy was stopped to get extreme hypotensive resuscitation strategy [8]. Hearth rate was 150beats/min, blood pressure $(\mathrm{BP})$ and peripheral oxygen saturation $\left(\mathrm{SpO}_{2}\right)$ was not measurable. Transferring the patient into the helicopter was logistically impossible and unsafe for the patient. Driving time by ambulance to the MTC was estimated in at least 20 minutes for a bank holiday traffic confirmed by HEMS crew flying to the scene. The estimated time of arrival to the nearest secondary acute care hospital identified as Spoke hospital in Romagna-SIAT was 8 minutes where 24/7 availability of emergency surgery and on call vascular team were confirmed (Figure 2). In the Spoke hospital no Trauma Team activation protocol was present, so that direct access to operating room (OR) was decided together with local anesthesiologist and surgeon by phone during ambulance transfer. Entering the OR, 2 anesthesiologists, 2 nurses and 1 surgeon were present. HEMS doctor, which was one of the Intensive Care doctors used to be involved in the MTC by Trauma Team activation, carried on Trauma Leader role. Early Coagulative Support Protocol started and mechanical ventilation carried on by Anesthesiologists [9]. Surgeon obtained Damage Control hemostasis with surgical forceps in few minutes and gradually the direct compression was freed. First available hemogasanalysis showed severe metabolic lactic acidosis with pH 7.009, Base Excess $-18 \mathrm{mmol} / \mathrm{L}$ and Lactate of $10.5 \mathrm{mmol} / \mathrm{L}$. About 50 minutes after 118 calling, bleeding was stopped. A total of $750 \mathrm{ml}$ of type 0 Rh negative packed red blood cells, 3 grams of Fibrinogen Concentrate and 2 grams of Tranexamic Acid were infused. Finally BP was $90 / 50 \mathrm{mmHg}$ and $\mathrm{SpO}_{2}$ was $100 \%$ with pulsed plethysmography. General anesthesia was maintained with ketamine, cis-atracurium and fentanyl. HEMS doctor left OR by delegating Trauma Leader role and HEMS begun operative again. Vascular Surgeons than could explore the injury, detecting right common carotid artery complete transection with lacerated stumps, right internal giugular vein complete transection, right sternocleidomastoid and subhyoid muscle complete transection, and right hemithyroid injury. Termino-terminal prosthetic bypass was performed to repair carotid artery and giugular vein (Figure 3), and other injuries was directly sutured. The patient was then transferred to Intensive Care Unit in stable hemodynamic and respiratory conditions and discharged by the hospital to rehabilitation center 22 days later. GCS was 15, breathing spontaneously, left hemiparesis due to cerebral right focal multiple ischemic lesions was present and documented injury to right brachial plexus, recurrent and phrenic ipsilateral nerves resulted in right harm, vocal cord and diaphragm paralysis.

\section{Conclusions}

According to literature primary referral to MTC of severely injured trauma victims is associated with better outcomes and HEMS is useful in a Trauma System to optimize timing and management during patient transfers to MTC. We presented a case in which massive bleeding in zone 1 of the neck made impossible the primary referral to MTC for clinical and logistical circumstances. The only available choice was to bring the patient to the nearest Spoke Hospital. Finally the optimal interaction between HEMS crew and Spoke Hospital personnel, used working with preferred partners, was crucial for good patient outcome in an unusual trauma scenario. To note that HEMS doctor attended just two weeks before the European Trauma Course that was extremely useful to take the Trauma Leader role performing with colleague not used to these situations [10].

\section{Declarations}

Compliance with ethical requirements: Ethics Approval and Consent to Participate Not applicable.

Consent for publication: Consent obtained directly from the patient, available for consultation.

Availability of data and supporting materials: Authors are available to share data and materials concerning the case report. 


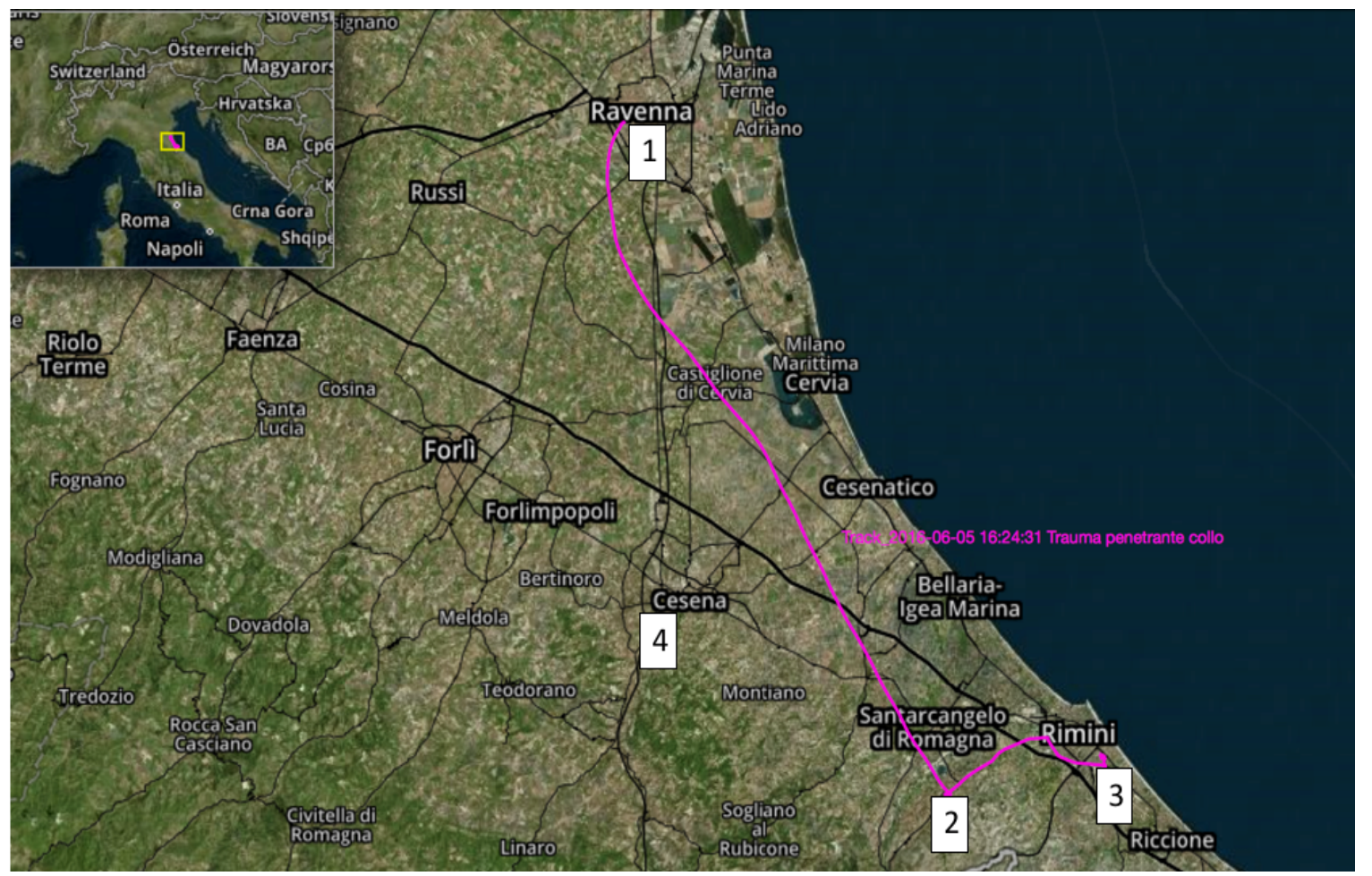

Figure 2. 1: HEMS base. 2: event scene. 3: spoke acute care "Infermi" hospital in Rimini. 4: Major Trauma Center "M. Bufalini" hospital in Cesena. 1-2 violet trace: flight trace to the scene. 2-3 violet trace: ambulance trace to Spoke "Infermi" hospital in Rimini.

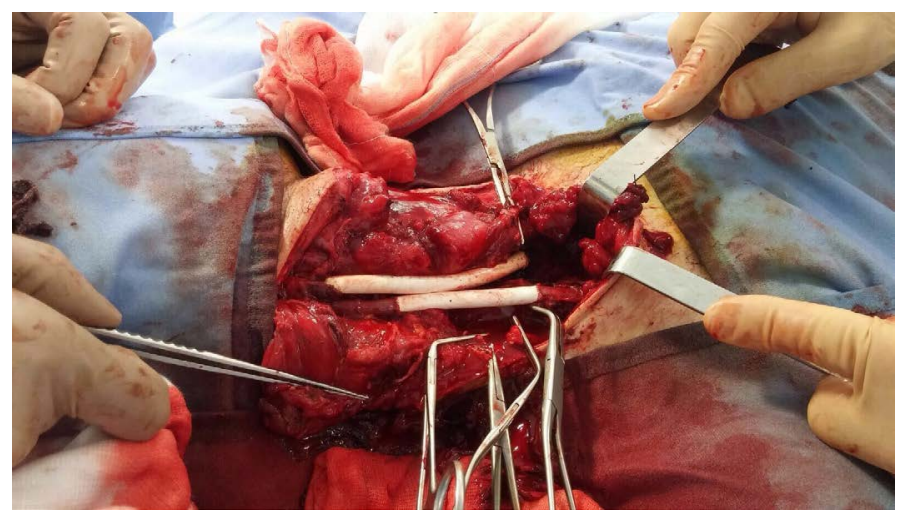

Figure 3. Intra-operative image of vascular prosthetic bypass repair.2-3 violet trace: ambulance trace to Spoke "Infermi" hospital in Rimini.

\section{Funding: None.}

\section{Conflict of interest statements}

Emiliano Gamberini, Antonela Potalivo, Marco Benni and Vanni Agnoletti declare that they have no conflict of interest.

\section{Author's contributions}

Emiliano Gamberini, Vanni Agnoletti: design and drafting of manuscript. Antonella Potalivo, Marco Benni: data retrieval; Vanni Agnoletti: writing and reviewing the final paper. Vanni Agnoletti, Marco Benni: collecting data and interpretation. All authors read and approved the final manuscript.

\section{Acknowledgments}

Not applicable.

\section{References}

1. McKee JL, Roberts DJ, van Wijngaarden-Stephens MH (2015) The right treatment at the right time in the right place: a population-based, before-and-after study of outcomes associated with implementation of an all-inclusive trauma system in a large Canadian province. Ann Surg 261: 558-564.

2. Nathens AB, Brunet FP, Maier RV (2004) Development of trauma systems and effect on outcomes after injury. Lancet 363: 1794-1801. [Crossref]

3. Taylor C, Jan S, Curtis K, Tzannes A, Li Q, et al. (2012) The cost-effectiveness of physician staffed helicopter emergency medical service (HEMS) transport to a major trauma centre in NSW, Australia. Injury 43: 1843-1849.

4. Larsen KT, Uleberg O, Skogvoll E (2010) Differences in trauma team activation criteria among Norwegian hospitals. Scand J Trauma Resusc Emerg Med 18: 21. [Crossref]

5. Petrie D, Lane P, Stewart TC (1996) An evaluation of patient outcomes comparing trauma team activated versus trauma team not activated using TRISS analysis. $J$ Trauma 41: 870-873. [Crossref]

6. Agenzia sociale sanitaria regionale, Regione Emilia-Romagna: La rete regionale dei SIAT.

7. Brywczynski JJ, Barrett TW, Lyon JA, Cotton BA (2008) Management of penetrating neck injury in the emergency department: a structured literature review. Emerg Med $J$ 25: 711-715.

8. National Institute of Health and Care Excellence (2016) Major trauma: Assestment and initial managment. National Institute for Health and Care Excellence: Clinical Guidelines (UK) Feb. [Crossref]

9. Nardi G, Agostini V, Rondinelli B, Russo E, Bastianini B, et al. (2015) Trauma-induced coagulopathy: impact of the early coagulation support protocol on blood product consumption, mortality and costs. Crit Care 19:83.

10. Thies KC, Deakin CD, Voiglio EJ, Rommens PM, Sabbe MB, et al. (2014) The European Trauma Course: trauma teaching goes European. Eur J Anaesthesiol 31: 1314. [Crossref]

Copyright: (C)2017 Gamberini E. This is an open-access article distributed under the terms of the Creative Commons Attribution License, which permits unrestricted use, distribution, and reproduction in any medium, provided the original author and source are credited. 